\title{
Harnessing Pivotal Advances for Production and Structural Derivation of the Promising Molecule Ursolic Acid
}

\author{
Hao-ran Liu ${ }^{1}$, Nadeem Ahmad ${ }^{1}$, Bo Lv ${ }^{1}$, and Chun $\mathrm{Li}^{1}$ \\ ${ }^{1}$ Affiliation not available
}

January 13, 2021

\begin{abstract}
Ursolic acid (UA) is a ursane-type pentacyclic triterpenoid compound, naturally produced in plants via specialized metabolism and exhibits vast range of remarkable physiological activities and pharmacological manifestations. Owing to significant safety and efficacy in different medical conditions, UA may serve as a backbone to produce its derivatives with novel therapeutic functions. This review systematically provides an overview of the pharmacological activities, acquisition methods and structural modification methods of UA. In addition, we focused on the synthetic modifications of UA to yield its valuable derivatives with enhanced therapeutic potential. Furthermore, harnessing the essential advances for green synthesis of UA and its derivatives by advent of metabolic engineering and synthetic biology are highlighted. In combination with the advantages of UA biosynthesis and transformation strategy, large-scale production and applications of UA is a promising platform for further exploration.
\end{abstract}

\section{Harnessing Pivotal Advances for Production and Structural Derivation of the Promising Molecule Ursolic Acid}

Hao-ran Liu ${ }^{\mathrm{a}}$ Nadeem Ahmad ${ }^{\mathrm{a}}$ Bo Lv ${ }^{\text {*** }}$ Chun Liabc*

1. Institute of Biochemical Engineering, School of Chemistry and Chemical Engineering, Beijing Institute of Technology, Beijing 100081, P.R. China

2. Key Lab for Industrial Biocatalysis, Ministry of Education, Department of Chemical Engineering, Tsinghua University, Beijing 100084, PR China

3. Center for Synthetic and Systems Biology, Tsinghua University, Beijing, 100084, China

Hao-ran Liu ${ }^{\mathrm{a}}$ Liuhr_bit@163.com

Nadeem Ahmad ${ }^{\mathrm{a}}$ dr.nadeem444@gmail.com

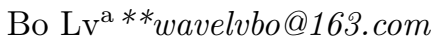

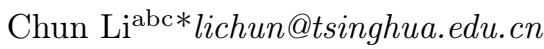

*Corresponding author. Institute of Biochemical Engineering, School of Chemistry and Chemical Engineering, Beijing Institute of Technology, Beijing 100081, P.R. China

** Corresponding author.

E-mail addresses: wavelvbo@163.com (B. Lv), lichun@tsinghua.edu.cn (C. Li).

Abstract: Ursolic acid (UA) is a ursane-type pentacyclic triterpenoid compound, naturally produced in plants via specialized metabolism and exhibits vast range of remarkable physiological activities and pharmacological manifestations. Owing to significant safety and efficacy in different medical conditions, UA 
may serve as a backbone to produce its derivatives with novel therapeutic functions. This review systematically provides an overview of the pharmacological activities, acquisition methods and structural modification methods of UA. In addition, we focused on the synthetic modifications of UA to yield its valuable derivatives with enhanced therapeutic potential. Furthermore, harnessing the essential advances for green synthesis of UA and its derivatives by advent of metabolic engineering and synthetic biology are highlighted. In combination with the advantages of UA biosynthesis and transformation strategy, large-scale production and applications of UA is a promising platform for further exploration.

Keywords: Ursolic Acid; Ursolic Acid derivatives; Biosynthesis; Metabolic engineering

Graphical abstract

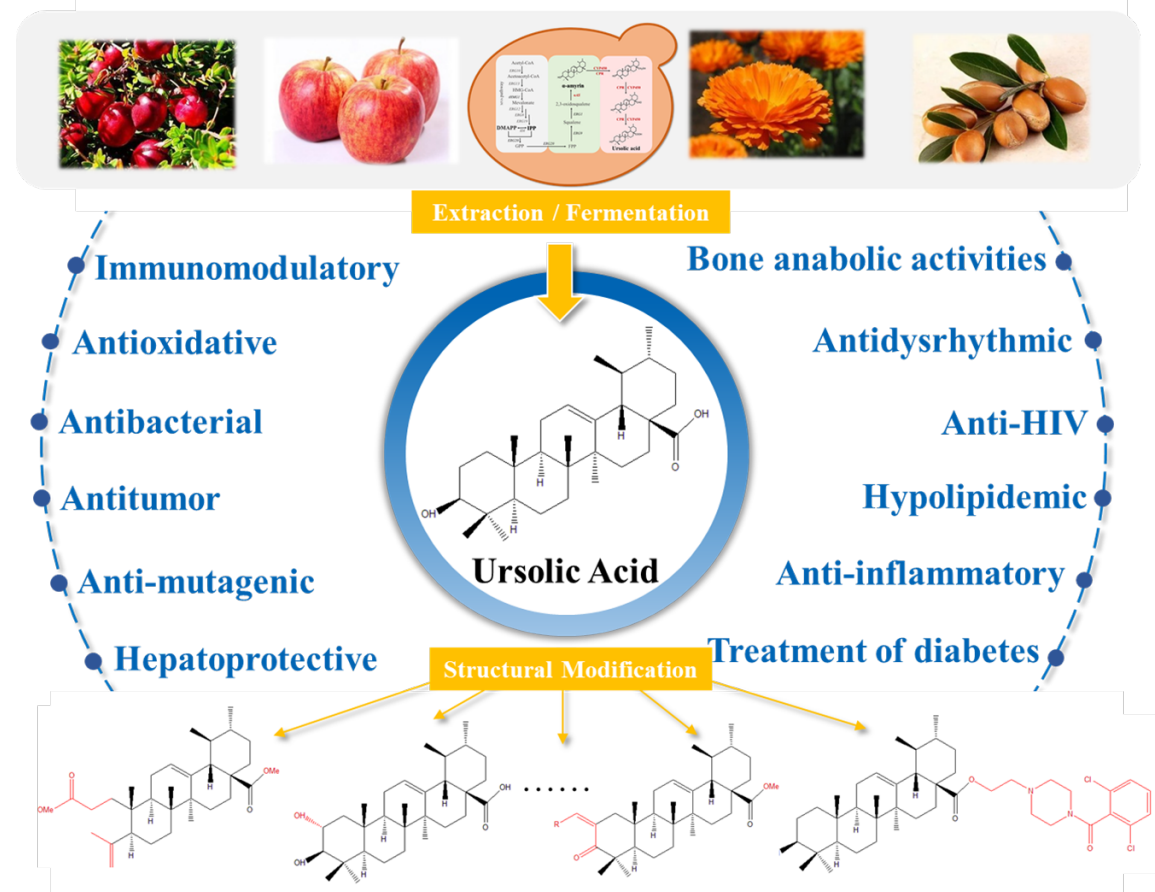

\section{Introduction}

Ursolic acid (UA, 33-hydroxy-urs-12-en-28-oic acid) is a kind of pentacyclic triterpenoids existing naturally in plants, which plays a significant role as a hormone or regulatory factor in plant growth and insect resistance ${ }^{1,2}$. Structurally, it is a ursane-type pentacyclic triterpenoid with C-3 hydroxy and C-28 carboxylic acid group (Fig. 1 ). Apart from its significance in plants, UA and its derivatives also exhibit remarkable physiological and pharmacological manifestations. Up to now, it has shown a wide range of clinical manifestations, including anti-inflammatory ${ }^{3}$, anti-oxidation ${ }^{4}$, diabetes treatment ${ }^{5}$, antibacterial ${ }^{6}$, anti-tumor metastasis ${ }^{7}$, anti-tumor activity ${ }^{8}$, anti-angiogenesis ${ }^{9}$, skin care $^{10}$, and superior liver protection function ${ }^{11}$.

Fig. 1 Chemical structure of Ursolic acid (UA)

The main way to obtain UA on a large scale currently relies on extraction of plants rich in it. However, it encounters several limitations, such as high energy consumption and low yield by plant extraction, which make it difficult to meet commercial needs. In addition, UA endures obstacles in therapeutic applications and drug development due to fast metabolism, poor water solubility and low bioavailability ${ }^{12,13}$.

In view of the excellent potential of UA as therapeutic drug, its structural modification is an attractive step to synthesize the potentially active derivatives in order to expand the pharmacological properties for 
further exploration ${ }^{14-16}$. More importantly, with the advent of synthetic biology and metabolic engineering, transition towards the synthesis of natural product by employing these approaches is of great concern. Cell factories such asSaccharomyces cerevisiae (S. cerevisiae) have been genetically engineered for green production of terpenoids, such as artemisinic acid ${ }^{17}$, ginsenoside ${ }^{18}$, oleanolic acid ${ }^{19}$ and lycopene ${ }^{20}$, which provide new thoughts for industrial production of UA. In this article, we systematically reviewed the significance of production and modification of UA to explore more applications in pharmacy and pharmaceutical chemistry. Particularly, we focused on the de novo synthesis of UA and its derivatives in engineered microbial cell factories in advancements for green production and green chemistry.

\section{Physiological and pharmacological profile of UA}

Studies have shown that UA has broad application prospects, which is employed in the management of various chronic diseases by mediating and regulating the pharmacological processes and related signaling pathways ${ }^{21,22}$.

UA plays an anti-inflammatory role in inflammatory cascade. It inhibits the activation, proliferation and cytokine secretion of $\mathrm{T}$ cells, B cells and macrophages of mouse lymphocytes. By inhibiting phosphorylation of Extracellular Regulated protein Kinases (ERK) and c-Jun N-terminal Kinase (JNK) induced by mitogen, it limits the activation of immunomodulatory transcription factors NF-kB, NF-AT and AP-1 in lymphocytes 23 .

In another study, UA has been revealed to exhibit hepatoprotective properties as it significantly inhibits the HCV genotype $1 \mathrm{~b}$ replicon and HCV genotype $2 \mathrm{aJFH} 1$ virus ${ }^{24}$. It is partly due to the inhibition of HCV-NS5BRdRp activity as a noncompetitive inhibitor. Researchers explored the expression of HPVE6/E7 by Reverse Transcription-Polymerase Chain Reaction (RT-PCR) and found that UA down-regulate it ${ }^{25}$.

UA also shown the promising results in the management of hepatitis A and B with the cure rate of $89.3 \%$ as it reduces the liver weight, ALT/AST level, hepatic steatosis, and palmitic acid induced fat accumulation in L02 cells $^{11,26}$. It exerts hepatoprotective effects by increasing $\beta$-oxidation of fat and inhibiting endoplasmic reticulum pressure.

In addition, UA exhibits significant therapeutic potential in cancer treatment due to its high activity and low toxicity. Studies have shown that UA decreases the activity of extracellular phosphorylated signal regulated kinase and depolarizes the mitochondrial membrane ${ }^{27}$. It has also been found that UA promotes the apoptosis of human bladder cancer cell line T24 by activating the ASK1-JNK signaling pathway and induction of the endoplasmic reticulum stress response ${ }^{28}$. Bioactivities of UA and its relevant mechanism of action have been summarized in Table 1 . On the basis of understanding pharmacological and physiological activities, UA will be better developed to meet more daily and medical needs.

Table 1. Bioactivities of UA and related mechanisms of action

\begin{tabular}{ll}
\hline Bioactivities & Mechanism of Action \\
\hline Antibacterial & effective on the biofilm of Gram-positive bacteria such as Staphylococcus aureus to prevent persistent \\
Anti-Angiogenesis & interference with Signal Transducer and Activator of Transcription 3 (STAT3), Mitogen-Activated P1 \\
Antihepatodamage & increase the $\beta$-oxidation of fat and inhibiting endoplasmic reticulum pressure. \\
Anti-Inflammatory & inhibits the phosphorylation of ERK and JNK, limits the activation of NF-KB, NF-AT and AP-1 in l \\
Antiviral & down-regulate HPVE6/E7 \\
Anti-Tumor & regulate transcription factors expression and protein kinases related to tumorigenesis. \\
Anti-Oxidation & regulates the changes of reactive oxygen species levels induced by ultraviolet B. \\
Diabetes Treatment & inhibits the activity of $\alpha$-amylase; reduces blood glucose levels and body mass index. \\
\hline
\end{tabular}

\section{How to obtain UA and what's challenges for application}

UA is widely distributed as triterpene saponin aglycone or free acid in various medicinal plants as well as sev- 
eral fruits, such asLigustrum lucidum ${ }^{33}$, Centella asiatica ${ }^{34}$, Lysimachia clethroides ${ }^{35}$, Crataegus pinnatifida ${ }^{36}$ and Malus domestica ${ }^{37}$. However, its percentage varies from species to species due to related biosynthetic enzymes and metabolism ${ }^{38}$. Currently, industrial production of UA mainly depends on extraction of natural plants. A variety of extraction methods have been developed to extract UA from different plants.

Table 2. Selected Methods for Isolation from Available Plant Sources for UA

\begin{tabular}{ll}
\hline Method & Extraction and purification \\
\hline Microwave Method & Weigh the plant powder and mix with the appropriate amount of extractant. After soaking \\
Rapid Solvent Extraction & The fast solvent method can be implemented using systems introduced by related compani \\
Impregnation & Wash the fruit and leaves with distilled water, then air dry for a week to remove all water. \\
Soxhlet Extraction & Degrease 4 parts of $2.00 \mathrm{~g}$ aliquots in Soxhlet extraction unit for 4 hours. After drying, $80 \%$ \\
Hot Reflux Method & The dried plant material was mixed with $100 \mathrm{~mL}$ of acetone. Connect with cooling water a \\
Ultrasonic Method & First, the ground material and solvent are mixed into the flask, and the flask is placed in a \\
Supercritical Fluid Extraction & First, the liquid carbon dioxide is pressurized to the required pressure in the coolant pump \\
\hline
\end{tabular}

However, plant cultures occupy a large area of cultivated land and plant growth may be affected by climate change and growth cycle, which subsequently reduces the adequate supply of raw materials. The challenges for UA's application also do include current production methods which consume a lot of organic reagents, and eventually produce waste liquids to cause serious ecological pressure to surrounding environment. So the traditional extractions of UA make it difficult to meet the commercial needs and environmental protection goals.

\section{Structure modification to improve the efficacy of UA}

Although UA exhibits remarkable physiological and pharmacological activities with effective and safe therapeutic profile, its poor water solubility, short plasma half-life and poor bioavailability limit further clinical applications ${ }^{44}$. In this regard, structural modifications of UA to cope with these limitations have been an attractive area of research to explore its valuable derivatives with enhanced pharmacological profile. In recent years, structural modification of UA is achieved by means of chemical transformation and microbial transformation to yield valuable derivatives.

\subsection{Chemical modification}

UA structure is characterized by a hydroxyl group and carboxylic group which can be involved in the transfer of lone pair electrons to metal atoms ${ }^{45}$. According to the structural properties of reported derivatives, UA usually undergoes structural modifications on C-3/C-28 positions or the ring A of UA skeleton.

\subsubsection{Modifications on $\mathrm{C}-3 / \mathrm{C}-28$}

Researchers isolated UA and five triterpenoids from apple peel and synthesized a series of UA analogs. Structural modification of UA at C-3 indicated significant anti-proliferative activity ${ }^{46}$. In another study, acetyl group at the C-3 and an alkylamino and/or piperidine moiety at the C-28 enhanced the anticancer activity of UA derivative ${ }^{46}$ (Fig. 2A ). In addition, structural modification of UA by addition of piperazine moieties may enhance its anti-cancer properties. Addition of acyl piperazine motif at C-28 position while C-3 retains the polar group significantly enhanced the anticancer activity against breast cancer and gastric cancer cell lines ${ }^{47}$ (Fig. $2 B$ ).

In order to clear on the mechanism of chemical modification at C-3 and C-28 in detail, researchers synthesized a series of UA derivatives by employing different electronic chemical modification at the two sites. Derivatives showed stronger cytotoxicity due to presence of positive charge (Fig. 2C ), indicating that the increased lipophilicity may enhance the therapeutic potential of gastric carcinoma ${ }^{48}$. These findings of UA derivatives led the ways to structurally modify the UA skeleton to yield its valuable derivatives with diverse pharmacological properties. 

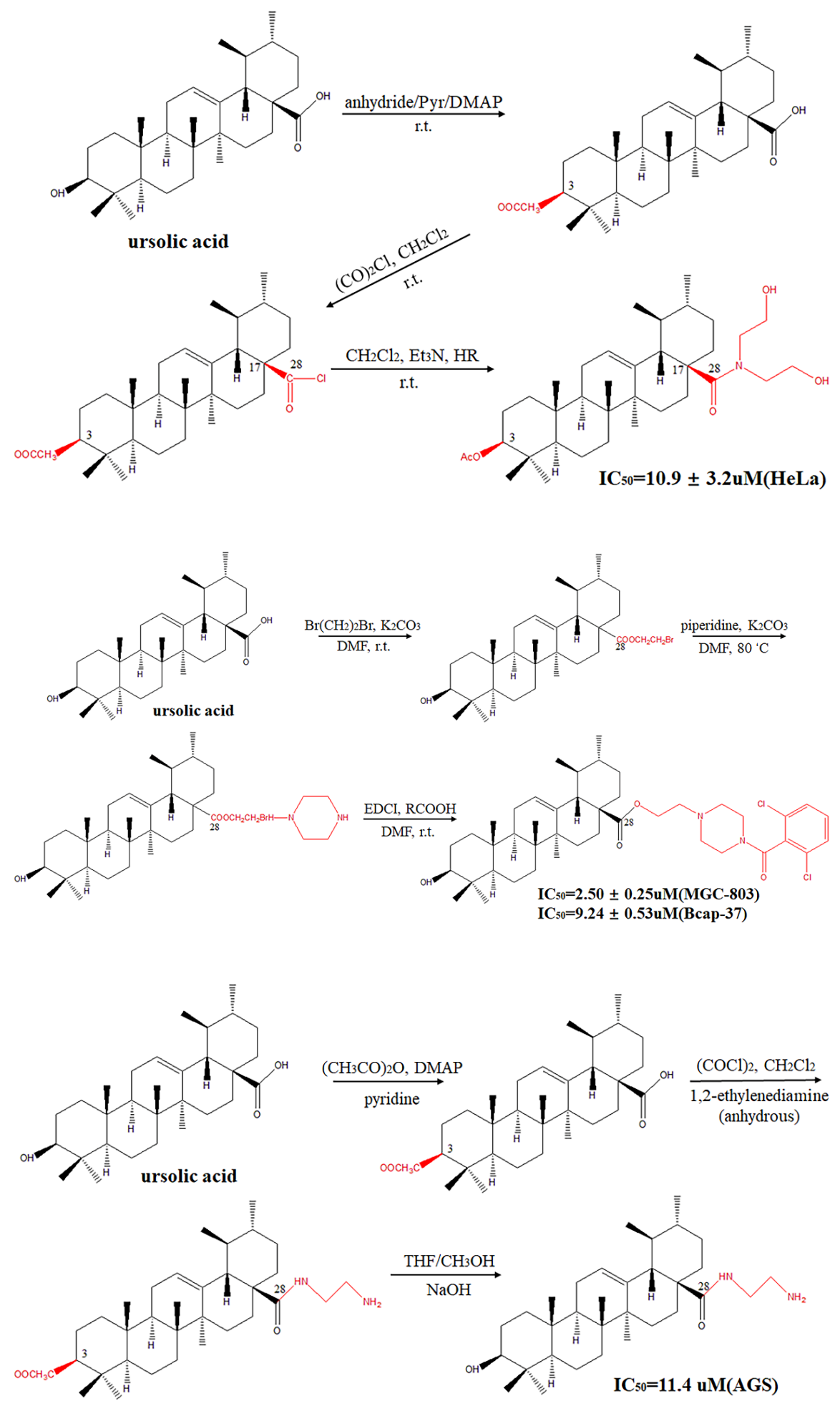

Fig. 2 Modification on C-3/C-28

\subsubsection{Modifications on ring A}


UA and Oleanolic acid (OA) are isomers, the difference between which is only at the position of Me29(Fig. 3 ). Derivatives of OA such as 2-cyano-3,12-dioxoolean-1,9-dien-28-oic acid (CDDO) exhibit significant antiinflammatory and anti-tumor activity due to the modification of the ring $\mathrm{A} 49,50$. Considering that both UA and OA are characterized by similar triterpene templates, similar chemical modifications have been applied to UA in recent years.

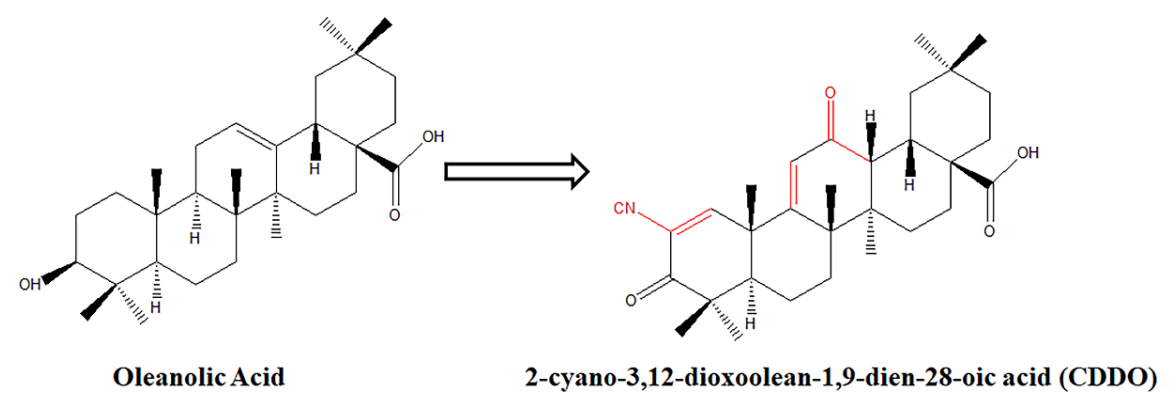

Fig. 3 Oleanolic Acid and CDDO

UA derivatives have been synthesized after oxidation, lactoseization and ring-opening reaction with increased cytotoxicity to NTUB1 cells compared to UA by 2-fold ${ }^{51}$ (Fig.4A), providing new insights for the further development of its derivatives. Moreover, UA derivatives containing $\mathrm{N}$-acyl imidazole or $\mathrm{N}$-alkyl imidazole derivatives with the help of $\alpha, \beta$-unsaturated ketone have also been characterized as significant anticancer activity ${ }^{52}$ (Fig.4B ). Introduction of $\mathrm{N}$-alkyl heterocyclic motifs bound to $\alpha, \beta$-unsaturated ketones in ring A can provide better Michael receptors, which allows UA derivatives to interact with certain target proteins interaction potentially.

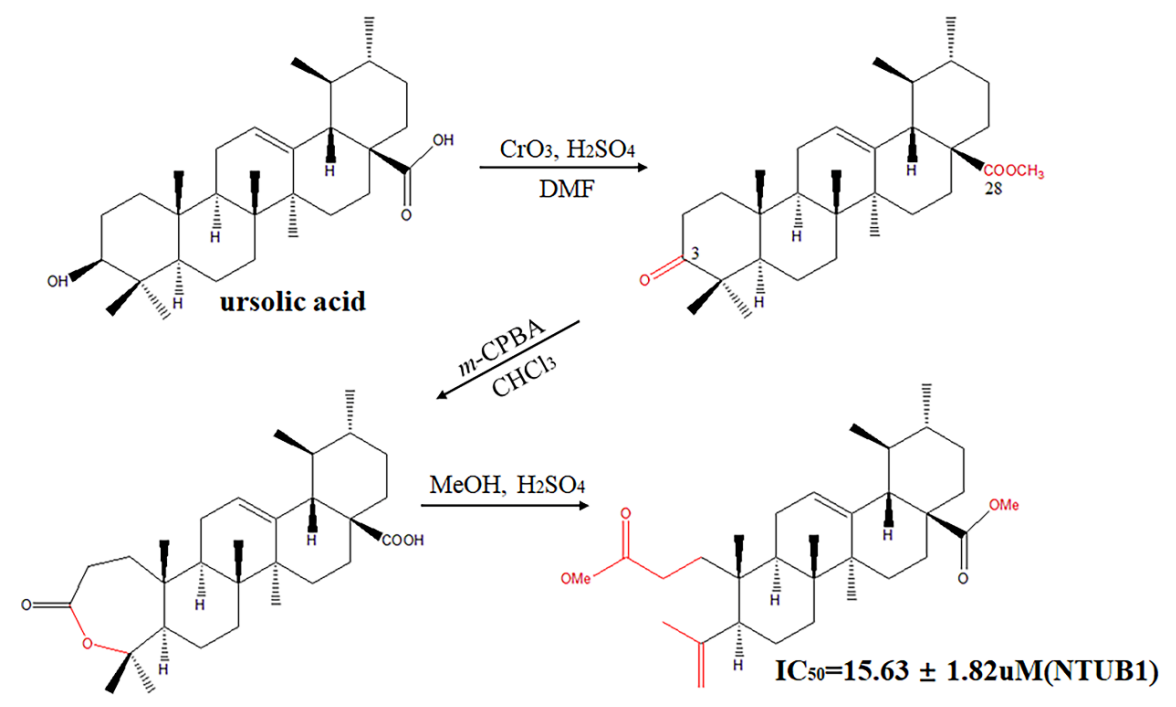




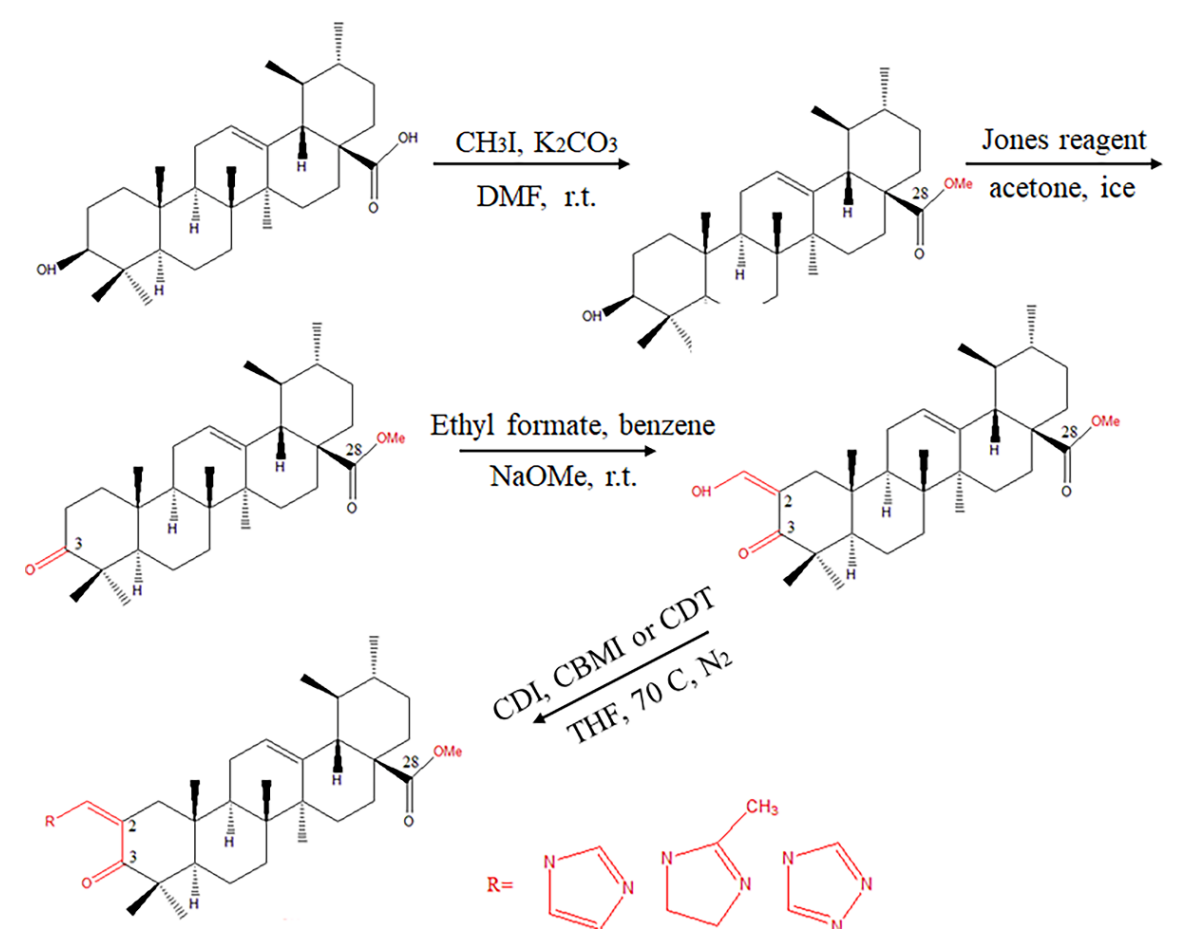

Fig. 4 UA derivatives modified on ring A

\subsection{Directed transformation of UA by microorganisms}

Biosynthesis of valuable products have advantages over chemical synthsis in terms of milder reaction conditions and presence of abundant enzymes along with high stereo-, regio- and chemo- selectivities ${ }^{53}$. Therefore, microbial transformation is increasingly adopted as an alternative way to modify the structure of natural and synthetic compounds ${ }^{54,55}$. In particular, it is easy to achieve the glycosylation of pentacyclic triterpenes that is difficult during chemical synthesis ${ }^{56}$. Generally, UA biotransformation is carried out by dissolving the appropriate quantity of UA in strain suspended in fresh sterile broth under sterile conditions. After cocultivation with strains, the transformed products of UA are eventually separated through suction filtration and extraction.

In recent years, various UA derivatives have been synthesized through the transformation of numerous microbial strains. It is reported thatNocardia sp. NRRL 5646 converts UA into oleanolic acid methyl ester (Fig.5A ), in which two intermediates are formed by participation in the "reverse biosynthesis" involving methyl migration from C-19 to C-20 ${ }^{57}$. A single component is produced by this biotransformation followed by hydrolysis to yield the corresponding triterpenoid acid. At the same time, UA has been found to be transformed into different derivatives by the same strain (Fig. $5 B$ ). Researchers speculate that $3 \beta$-hydroxysteroid dehydrogenase and 3-ketosteroid- $\Delta 1$-dehydrogenase are involved in the transformation of steroid ${ }^{58}$. 


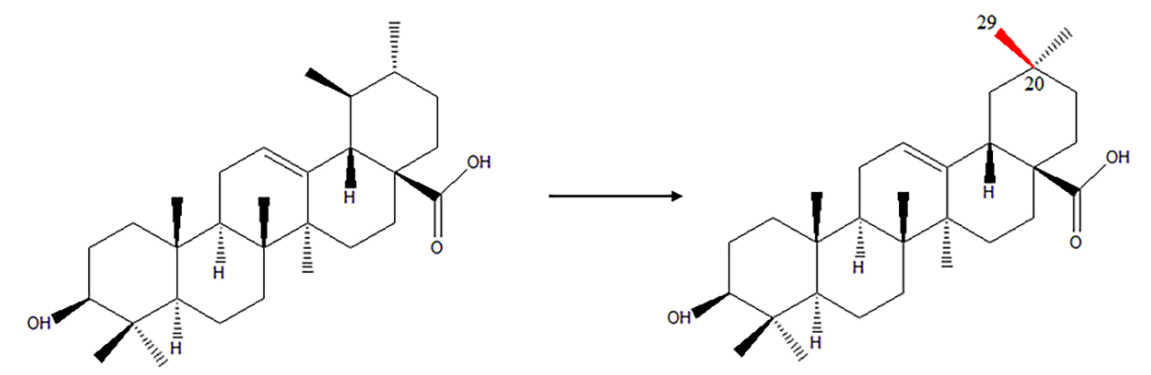

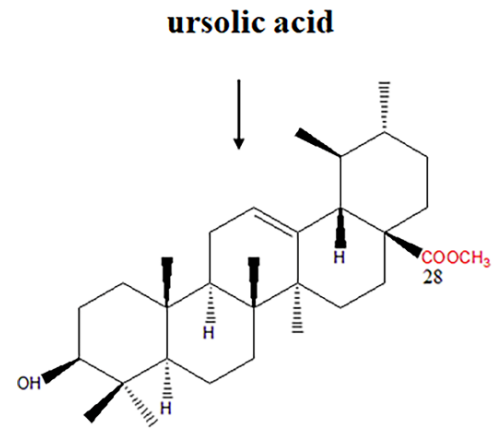

ursolic acid methyl ester

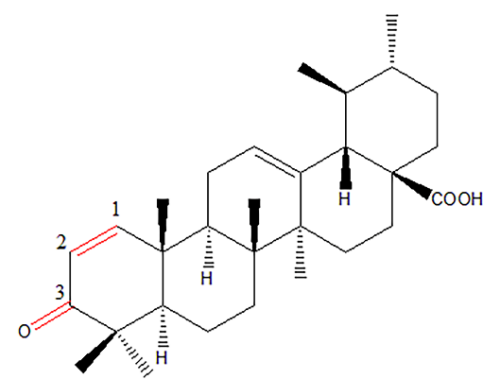

3-Oxoursa-1,12-dien-28-oic acid

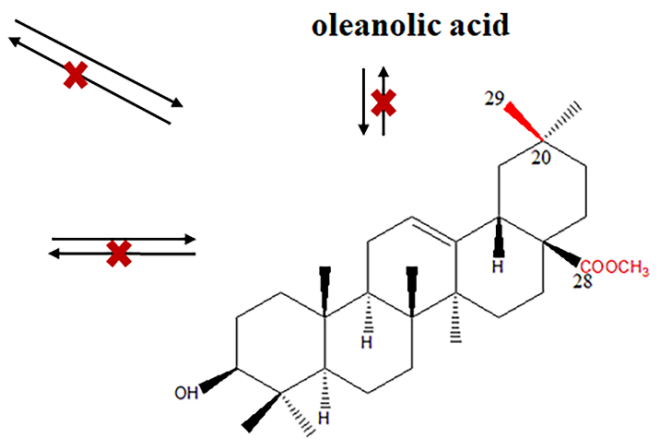

oleanolic acid methyl ester

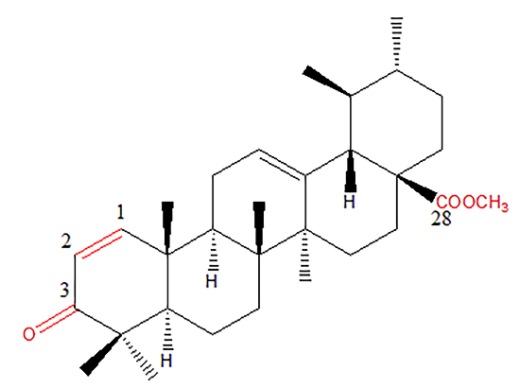

3-Oxoursa-1,12-dien-28-oic acid methyl ester

Fig. 5 Transformation of UA by Nocardia sp. NRRL 5646

Moreover, UA has been well modified by various fungi ${ }^{59}$. Aspergillus flavus (ATCC 9170) was able to convert UA into the new natural product 3-oxo-UA derivative with C-3 hydroxylation (Fig. 6A) ${ }^{60}$. In addition, four UA derivatives have been produced by Syncephalastrum racemosum(Cohn) Schroter AS3.264 ${ }^{61}$, indicating the inhibitory effect against protein tyrosine phosphatase 1B (Fig. 6C). UA was also biotransformed into six glycosylated and hydroxylated products by an endophytic fungi Alternaria longipes AS3.2875 ${ }^{62}$. One of the resultant new compounds, 28-o- $\beta$-d-glucopyranosyl-3-oxo-UA, showed stronger antibacterial activity against Staphylococcus aureus and methicillin-resistantStaphylococcus aureus (Fig. 6B ). Liu et al.obtained three

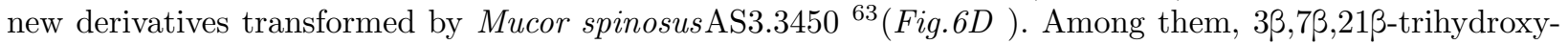
UA showed stronger cytotoxicity against HeLa cells, K562 cells and KB cells, enlightening its potential therapeutic role in anticancer therapy. 


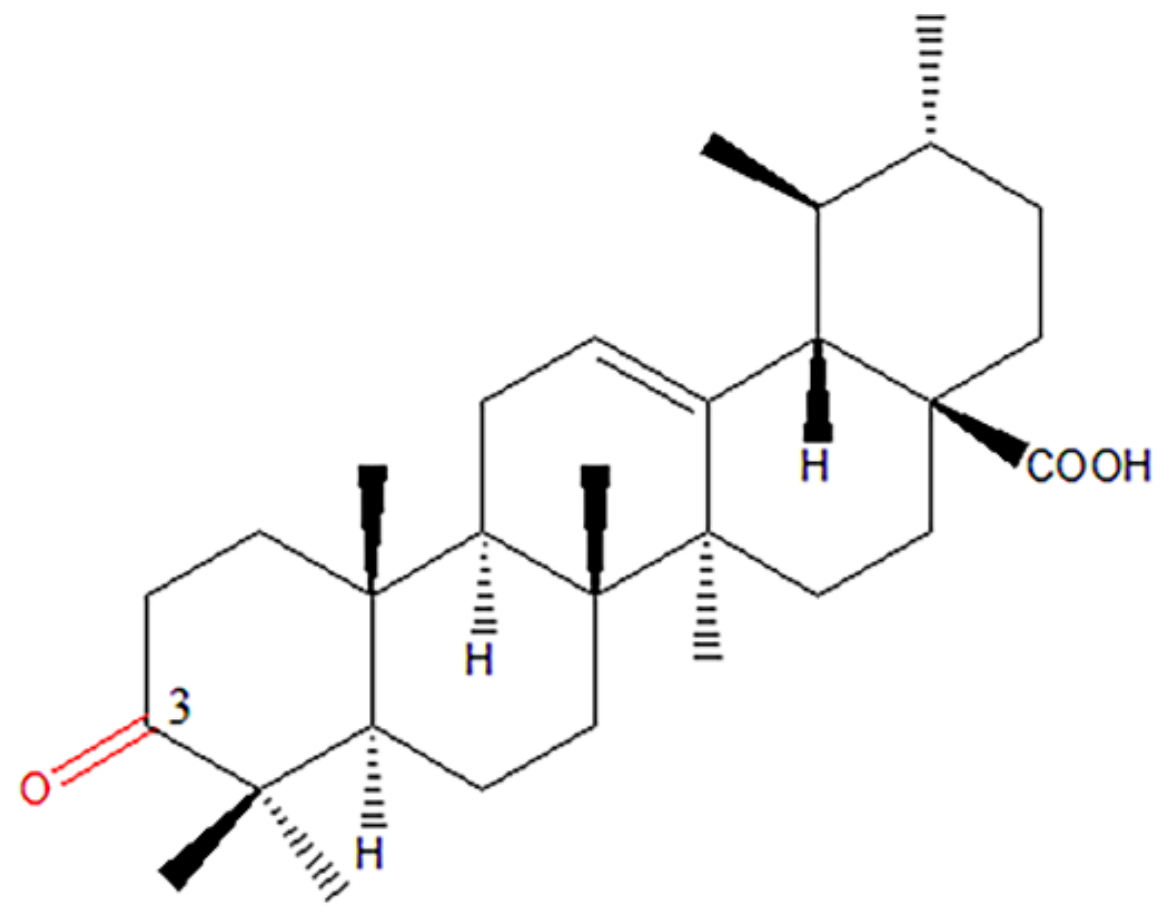

3-oxo derivative of ursolic acid

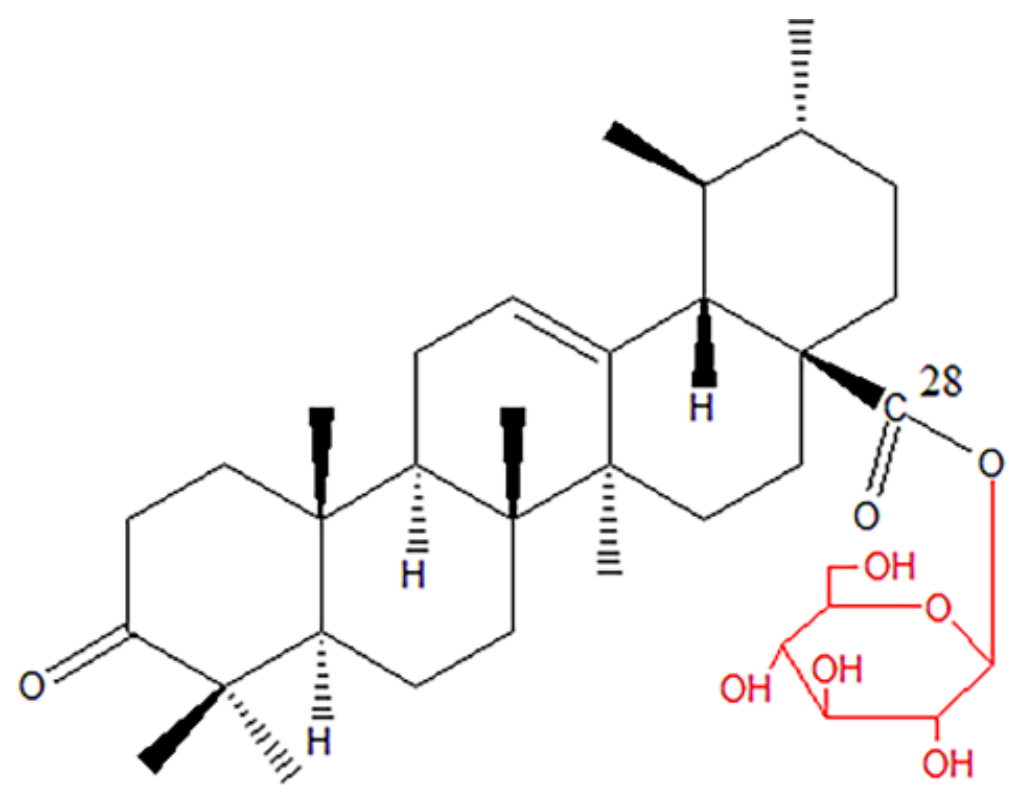

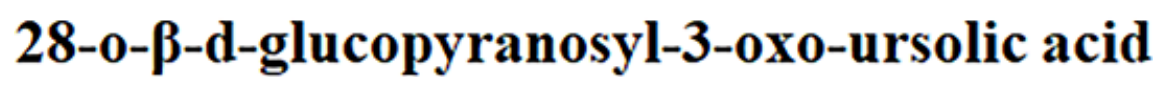




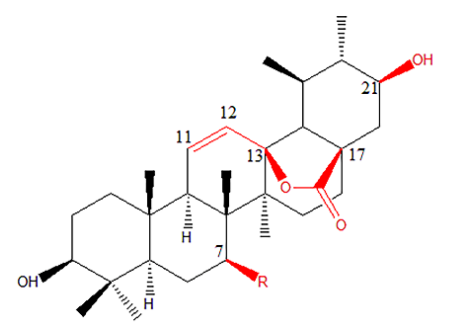

3b,21b-dihydroxy-urs-11-en-28-oic acid-13-lactone

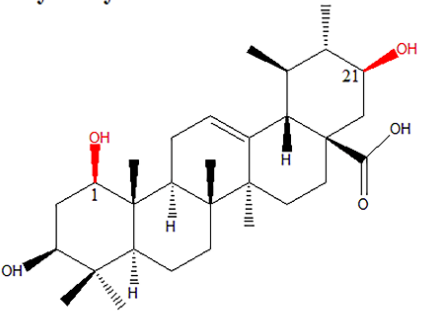

1b,3b,21b-trihydroxy-urs-12-en-28-oic acid

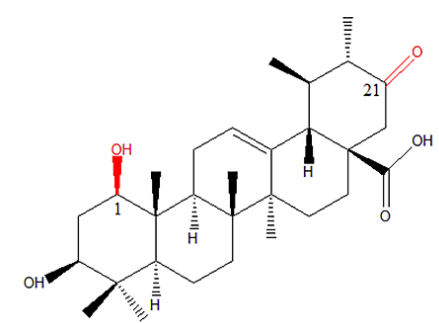

1b,3b-dihydroxy-urs-12-en-21-one-28-oic acid

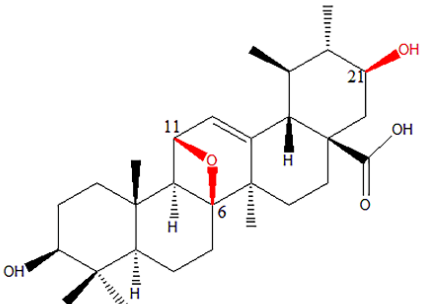

11,26-epoxy-3b,21b-dihydroxy-urs-12-en-28-oic acid

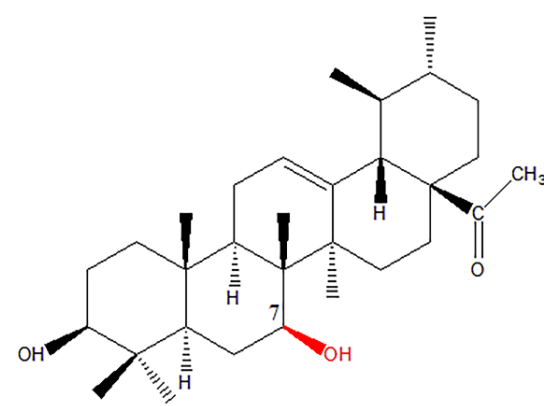

$3 \beta, 7 \beta$-dihydroxy-ursolic acid-

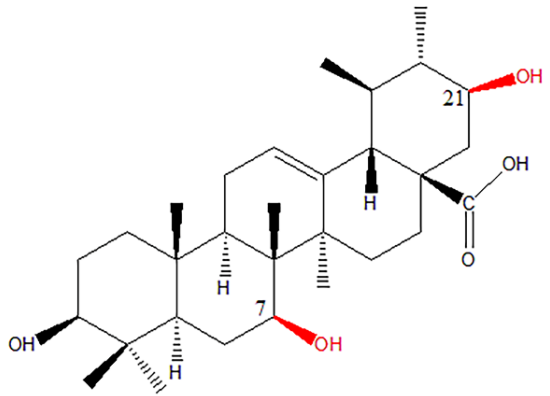

$3 \beta, 7 \beta, 21 \beta$-trihydroxy-ursolic acid 28-ethanone

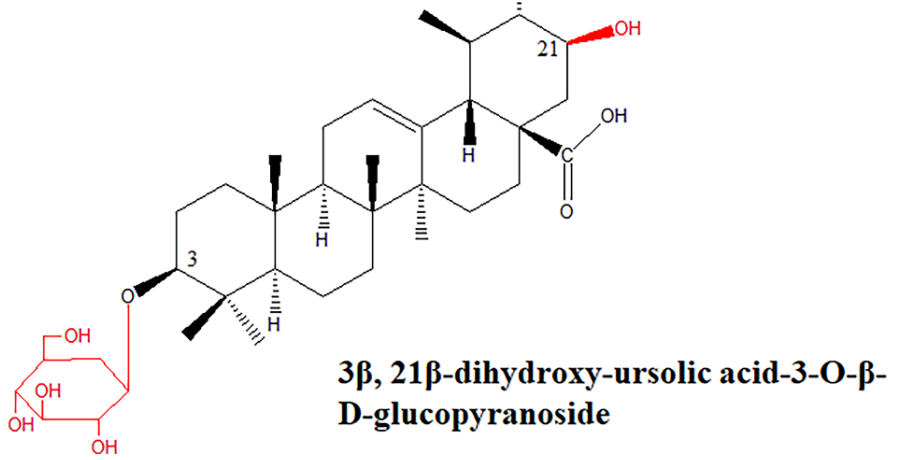

Fig. 6 UA derivatives biotransformed by fungi

\section{Efficient synthesis of UA and its derivatives in engineered microbial cell factories}

As mentioned above, traditional extraction methods are increasingly unfavorable for large-scale production of UA. Furthermore, complex structure and oxidation position, as well as the optical purity of the final product all increase the difficulty for its chemical synthesis. Green biosynthesis of valuable compounds by microbial cell factories may reduce the limitations of traditional extraction methods from plants as it is not restricted by the natural climate and is suitable for large-scale industrial production under high density 
fermentation. More importantly, some terpenoids are synthesized efficiently in microorganisms with various regulatory strategies, such as excavation and modification of key enzymes ${ }^{18,64}$, optimization of endogenous metabolic pathways ${ }^{65-67}$, inhibition of branching pathways ${ }^{68-70}$. Therefore, microbial engineering also has a wide application prospect in the green synthesis of UA and its derivatives.

\subsection{The biosynthesis pathway of UA}

In recent years, synthetic pathway of UA has been well explored in plants, which is roughly divided into three modules: synthesis of isopentenyl diphosphate (IPP) and its isomer dimethylallyl diphosphate (DMAPP), synthesis of $\alpha$-amyrin and oxidation of $\alpha$-amyrin. In plant organelles, IPP and DMAPP are synthesized by methyl erythritol-4-phosphate (MEP) pathway in plastids and mevalonate (MVA) pathway in cytoplasm. Afterwards, two IPP molecules are combined with one molecule of DMAPP end-to-end, and condense to farnesyl diphosphate (FPP). Two FPP molecules bind together to form the triterpenoid precursor, squalene followed by oxidiation to form 2,3-oxidosqualene, which subsequently undergoes cyclization into $\alpha$-amyrin, the direct precursor of UA. Eventually, $\alpha$-amyrin is oxidized at C-28 to form UA through a three-step oxidation reaction by employing the action of Cytochrome P450 (CYP450) monooxygenase and its redox chaperone Cytochrome P450 Reductase (CPR). In addition to the common rate-limiting enzymes for triterpenoid, $\alpha$-amyrin synthase $(\alpha \mathrm{AS})$ and CYP450 greatly affect the synthesis of UA (Fig. $7 A$ ).

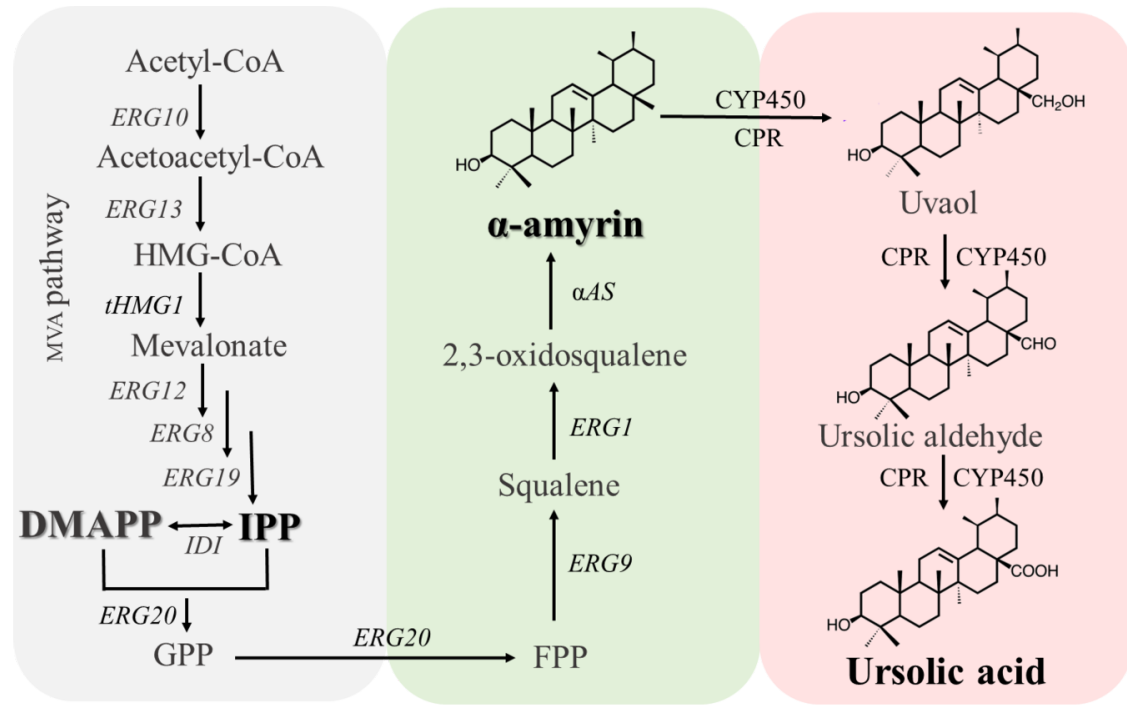




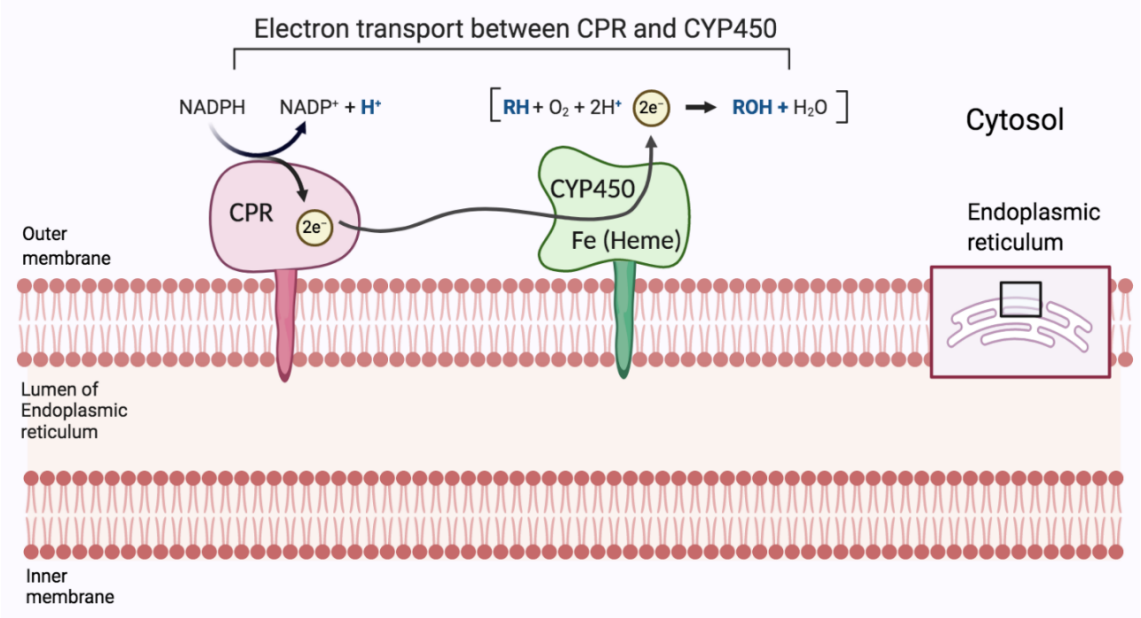

Fig. 7 Biosynthesis of UA (A) Biosynthesis pathway; (B) The electron transfer between CYP450 and CPR

It is generally believed that cyclization of 2,3-oxidosqualene is carried out by the Oxidosqualene Cyclase (OSC) by employing protonation, cascade cyclization of polyene addition, hydride and/or methyl translocation, and unwinding ${ }^{71}$. $\alpha$ ASs are multifunctional enzymes, which catalyze 2,3 -oxidosqualene to several pentacyclic triterpenoids like $\alpha$-amyrin, $\beta$-amyrin and lupeol etc ${ }^{72,73}$. However, product isomerization limits their catalytic ability and ultimately affects the downstream synthesis of UA. CYP450s are most versatile proteases found in nature, the first one of which was discovered in the rat liver microsomes in $1958^{74}$. CYP450s contain the heme, which primarily binds to the cytoplasmic surface of Endoplasmic Reticulum (ER) and catalyze a series of oxidation reactions like oxidation, hydroxylation, dealkylation and breakage of carbon-carbon bonds ${ }^{75}$. Mainly, CYP450s catalysis involves the introduction of oxygen into inactive carbon-hydrogen bonds ${ }^{76}$, while the corresponding reduction process is assisted by CPR. CYP450s utilize two electrons from NADH or NADPH transferred by CPR to the heme center of iron to activate the oxygen molecules and further catalyze the substrates to form functional groups.

\subsection{De novo synthesis of UA in engineered microbial cell factories}

With strong resistance to low $\mathrm{pH}$, osmotic stress and environmental factors, engineered microbial cell factories such as $S$. cerevisiae is widely used to biosynthesize the valuable natural products on industrial scale. What's more, $S$. cerevisiae has proven to be an enormously suitable candidate for synthesis of complex terpenoids due to its endogenous MVA pathway, which provides sufficient precursors to synthesize the terpenoids to meet subsequent demand ${ }^{65}$. Terpene skeleton is modified by CYP450 in a better way due to presence of protein modification system, biofilm system and redox system in S. cerevisiae ${ }^{18}$. With clear genetic background and suitable biological safety, various gene manipulation and genetic modification methods have been widely employed in S. cerevisiaefor the green synthesis of terpenoids.

As early as 2012, $\alpha$-amyrin has been synthesized in microbial cell factories ${ }^{77}$, but the UA synthesis was inadequate. It is generally accepted that UA biosynthesis in S. cerevisiae is limited due to the accumulation of $\alpha$-amyrin and the oxidation ability of CYP450 ${ }^{78}$. These limitations led researchers to adopt metabolic engineering and synthetic biology approaches to synthesize UA more efficiently in S. cerevisiae. Dai et al. introduced CYP716A15 and CPR from Vitis vinifera and $\alpha \mathrm{AS}$ fromEriobotrya japonica into S. cerevisiae . With ERG1, ERG 9 and ERG 20 overexpressed to enhance the precursor production, they subsequently got a final UA yield of $1.76 \mathrm{mg} / \mathrm{L} / \mathrm{OD}_{600}{ }^{75}$. In another study, Lu et al. heterologously expressed CYP716A12 from Medicago Sativa, aAS fromCatharanthus roseus, ERG1 from Candida albicans, and CPR from Arabidopsis thaliana in Saccharomyces cerevisiae. By optimization of fermentation conditions and overexpression of tHMG1, ERG9, ERG20 to strengthen MVA pathway, the yield of UA in shake flask culture 
reached $25.85 \mathrm{mg} / \mathrm{L}^{79}$.

\subsection{Optimization strategies to enhance the microbial synthesis of UA}

Biosynthesis of UA in microbial cell factories provides an environment friendly platform for synthesis of specialized terpenoids, while the bioproduction of UA still needs to be improved as compared to its counterpart triterpenes. Therefore, multiple optimization strategies are still needed to further develop the production potential of $S$. cerevisiae. On the basis of UA's synthetic pathway, we propose three strategies to enhance the green biosynthesis of UA at industrial scale.

\subsubsection{Strengthening precursors supplementation}

Microbial biosynthesis of natural products is improved by adopting effective metabolic engineering approaches to enhance the precursors supply and reducing their unnecessary consumption. Common strategy includes the overexpression of MVA-related (e.g. ERG9, ERG1and ERG20) genes and tHMG1 (the truncated version of HMG1) gene. Lu et al.enhanced the supply of 2,3-oxidosqualene as mentioned above, resulting in 25.99 $\mathrm{mg} / \mathrm{L} \alpha$-amyrin in shake flask culture ${ }^{79}$.

Enhancement of precursor supply is also achieved by adopting the traditional metabolic engineering approaches to overexpress the key genes, utilization of balanced pathways and downregulation of competitive pathways ${ }^{80}$. In another study, researchers balanced the metabolic pathway and achieved transcriptional regulation of aligned oleanane-type triterpenoids by overexpression of UPC2-1, a global transcription factor for ergosterol synthesis in yeast ${ }^{81}$. In addition, they reconstructed the promoter at the binding site of UPC2 and the galactose regulatory network to promote gene expression, resulting in a 65 and 6.8 -fold increase in $\beta$-amyrin and oleanolic acid, respectively ${ }^{82}$. This also provides a new idea for the synthesis and regulation of both $\alpha$-amyrin and UA in yeast.

Furthermore, the catalytic potential of $\alpha \mathrm{AS}$ also plays a decisive role in the synthesis of $\alpha$-amyrin and UA. A highly active $\alpha \mathrm{AS}, M d$ OSC1 from Malus domestica, have been identified through bioinformatics screening and phylogenetic analysis ${ }^{37}$. Md OSC1 expression combined with overexpression of genes related to the MVA pathway in $S$. cerevisiae yielded an $\alpha$-amyrin titer of $11.97 \pm 0.61 \mathrm{mg} / \mathrm{L}^{83}$. Furthermore, the yield of $\alpha$-amyrin was increased to 11-fold higher than that of the control with the triple mutant $M d$ OSC1 ${ }^{\mathrm{N} 11 \mathrm{~T} / \mathrm{P} 250 \mathrm{H} / \mathrm{P} 373 \mathrm{~A}}$ by remodeling $M d \mathrm{OSC}^{84}{ }^{84}$. Owing to these strategy, UA biosynthesis will be guaranteed by enhancing the supply of its precursors.

\subsubsection{Enhancing the coupling efficiency of CPR with CYP450}

Lower microbial production of UA as compared to $\alpha$-amyrin depicts the inefficient oxidation process which in turn depends upon both the catalytic efficiency of CYP450 as well as electron transfer between CYP450 and $\mathrm{CPR}^{80}$. In this regard, it is essential to excavate and characterize CYP450 and CPR with high catalytic efficiency. By means of gene linkage, genome sequencing and transcriptome sequencing, many CYP450s with the ability to oxidize C-28 of $\alpha$-amyrin have been identified from Arabidopsis thaliana, Medicago truncatula , Barbarea vulgaris and other plants. Combining different sources of CYP with CPR and finding the best combination is a common way to improve its oxidation ability (Table 3 ).

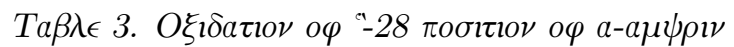

\begin{tabular}{lll}
\hline CYP450 & Source & Function \\
\hline CYP716A1 & Arabidopsis thaliana & C-28 oxidation of $\alpha$-amyrin and $\beta$-amyrin; C-28 hydroxylation of lupeol \\
CYP716A2 & Arabidopsis thaliana & C-28/C-22 $\alpha$ hydroxylation of $\alpha$-amyrin; C-16/C-22 $\alpha /$ C-28 hydroxylation of $\beta$ - \\
CYP716A12 & Medicago truncatula & C-28 oxidation of $\alpha$-amyrin, $\beta$-amyrin and lupeol \\
CYP716A15 & Vitis vinifera. & C-28 oxidation of $\alpha$-amyrin, $\beta$-amyrin and lupeol \\
CYP716A44 & Lycopersicum esculentum & C-28 oxidation of $\alpha$-amyrin, $\beta$-amyrin \\
CYP716A46 & Lycopersicum esculentum & C-28 oxidation of $\alpha$-amyrin, $\beta$-amyrin \\
CYP716A48 & Oleaeuropaea. & C-28 oxidation of $\alpha$-amyrin, $\beta$-amyrin and lupeol
\end{tabular}




\begin{tabular}{lll}
\hline CYP450 & Source & Function \\
\hline CYP716A49 & Beta vulgaris. & C-28 oxidation of $\alpha$-amyrin, $\beta$-amyrin and lupeol \\
CYP716A50 & Coffea arabica. & C-28 oxidation of $\alpha$-amyrin, $\beta$-amyrin and lupeol \\
CYP716A52V2 & Panax schinseng & C-28 oxidation of $\alpha$-amyrin, $\beta$-amyrin and lupeol \\
CYP716A80 & Barbarea vulgaris & C-28 oxidation of $\alpha$-amyrin, $\beta$-amyrin and lupeol \\
CYP716A81 & Barbarea vulgaris & C-28 oxidation of $\alpha$-amyrin, $\beta$-amyrin and lupeol \\
CYP716A83 & Centella asiatica & C-28 oxidation of $\alpha$-amyrin, $\beta$-amyrin \\
CYP716A175 & Malus domestica & C-28 oxidation of $\alpha$-amyrin, $\beta$-amyrin, lupeol and germanicol \\
CYP716A179 & Glycyrrhiza uralensis & C-28 oxidation of $\alpha$-amyrin, $\beta$-amyrin and lupeol \\
CYP716A252 & Ocimum basilicum & C-28 oxidation of $\alpha$-amyrin, $\beta$-amyrin \\
CYP716A253 & Ocimum basilicum & C-28 oxidation of $\alpha$-amyrin, $\beta$-amyrin \\
CYP716A265 & Lagerstroemia speciosa. & C-28 oxidation of $\alpha$-amyrin, $\beta$-amyrin and lupeol \\
CYP716A266 & Lagerstroemia speciosa. & C-28 oxidation of $\alpha$-amyrin, $\beta$-amyrin and lupeol \\
CYP716AL1 & Catharanthus roseus & C-28 oxidation of $\alpha$-amyrin, $\beta$-amyrin and lupeol \\
\hline
\end{tabular}

In addition, exact mechanism of action of CYP450 and CPR is not clear yet and there is a need to explore the ultimate cause that affects the electron transfer efficiency between them. Analysis of the protein structure of CYP450 helps to elucidate the underlying causes of electron transfer from CPR to CYP450. In addition, with the advent of protein engineering along with high throughput screening, rational design and modification of CYP450 and CPR have attracted much attention, which with optimized structures have also become common means to solve the problem of insufficient oxidation ${ }^{80}$.

\subsubsection{Optimization of subcellular structure}

S. cerevisiae harbors diverse organelles with different structures and specialized functions. Rational design and harnessing of these organelles to produce valuable compounds is of great application prospect in terpenoid synthesis. Heterologous FPP synthase and sesquiterpene synthase to mitochondria by fusing the mitochondrial targeting signal peptide of yeast COX4, resulting in a 20 -fold increase in sophoradiene production ${ }^{98}$. ER can also be subjected to morphological modifications such as increasing the membrane area to harbor more enzymes to enhance the catalysis processes. As many endogenous or exogenous proteins are located in ER membrane, like CYP450, the area of ER membrane have been significantly increased by knocking out the PAH1 by CRISPR/cas9 in S. cerevisiae to enhance the activity of enzymes related to terpene synthesis for efficient biosynthesis of terpenoids ${ }^{99}$. This engineering strategy increased the yield of $\beta$-amyrin, artemisinic acid, alfalfa acid and its glycosylated derivatives by 8 -fold, 2-fold, 6-fold and 16-fold respectively, which showed great potential of microbial cell factories to enhance the yield of terpenoids.

In addition, mass transfer efficiency can significantly boost the synthetic capacity of microorganisms ${ }^{65}$. The highest yield of $\alpha$-amyrin was obtained in engineered $S$. cerevisiae by expanding the storage pool, where DGA1 (Diacylglycerol acyltransferase) was overexpressed to enhance the intracellular storage capacity ${ }^{84}$. Draw lessons from it, UA biosynthesis can also be improved by modifying chemical mass transfer such as the translocation of its synthesis and the condition of transportation.

By combining all these strategies including the precursor supplementation, enhancing the coupling efficiency of CPR with CYP450 and optimization of subcellular structure, de novo biosynthesis of UA will be significantly enhanced in microbial cell factories. These studies fully demonstrated the potential of utilizing S. cerevisiae cell factories to synthesize UA efficiently, providing an effective means to replace traditional extraction and chemical synthesis.

\subsection{UA derivatives decorated by engineered microorganisms}

Biosynthesis of UA derivatives by construction of metabolic pathways in engineered microbial cell factories have been promoted along with the development of de novo microbial synthesis of UA. In recent years, biosynthesis of UA derivatives mainly involved in hydroxylation on C- $2 \alpha$ and glycosylation. 
For C-2 $\alpha$ hydroxylation of UA, sequence analysis of RNA of Avicennia marina leaves revealed the functional CYP716C53, which catalyzed the C-2 $\alpha$ hydroxylation of UA to yield Corosolic acid (Fig. 8 ) ${ }^{100}$. It's a triterpenoid compound which has attracted commercial and research interest for unique anti-diabetic properties ${ }^{101}$. In addition, the heterologous expression of Lagerstroemia speciose-CYP716C55 in S. cerevisiae also led to C-2 $\alpha$ hydroxylation of UA ${ }^{96}$. Moreover, CYP716C49 was identified in Crataegus pinnatifida and its expression along with the $\alpha \mathrm{AS}$ from E. japonica as well as CYP716A15 and CPR from V. vinifera in microbial cell factories increased the production titer of Corosolic acid to $141.0 \mathrm{mg} / \mathrm{L}^{75}$.

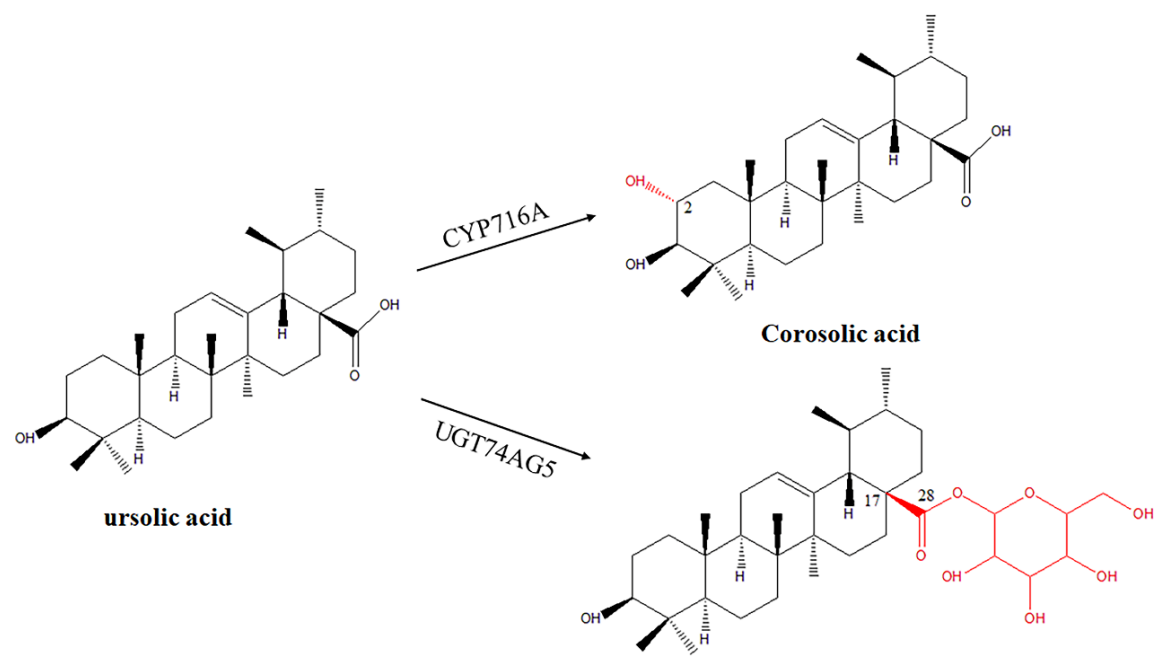

ursolic acid 28-O- $\beta$-D-glucopyranoside

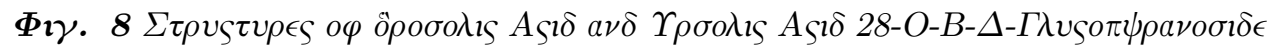

Glycosylation involves the attachment of one or more sugar moieties to the parent compound and contributes for diverse physicochemical properties, such as water solubility, structural integrity and pharmacological efficacy ${ }^{80}$. To achieve the glycosylated derivatives, heterologous expression of UDP-glycosyltransferases (UGTs) is less frequent due to their lower in vivo catalytic activity. In relation to glycyrrhetinic acid, structural diversification of UA by glycosylation is still in infancy. In one study, transcriptomic data analysis of Ilex Asprella revealed UGT74AG5 and its expression in UA-producing $S$. cerevisiae strain confirmed its glycosylation activity against UA to synthesize its glycosylated derivative, UA-28-O- $\beta$-D-glucopyranoside ${ }^{102}$ (Fig. 8 ). Although the synthesis of UA derivatives in cell factories is still in its infancy, the de novo synthesis of UA in S. cerevisiae provides strong support for its development.

\section{Perspective}

UA exhibits significant therapeutic properties to be used in disease management and drug development. UA derivatives with different structural modifications have been designed and synthesized to explore more effective therapeutic products with higher oral bioavailability and efficacy. Particularly, many structurally modified UA derivatives by chemical means seem to be effective against variety of cancer cell lines in vitro. Due to the great value potential and pharmacological manifestations of UA and its derivatives, there is a need to develop an economical and feasible approach to produce the high yield of UA and its varied derivatives.

Although considerable efforts are currently being made to develop effective approaches for sequestering UA from various medicinal plants, its biosynthesis in microbial cell factories is a more attractive strategy. In this regard, S. cerevisiae presents a potential microbial host to synthesize terpenoids efficiently. However, bioengineering strategies for bioproduction of either UA or its derivatives have not been developed effectively. With the advent in synthetic biology and metabolic engineering, improved metabolic pathways and key enzyme-protein engineering lead to efficient biosynthesis of valuable products, which provides strong support 
for better utilizing $S$. cerevisiae as a cell factory. Increased production of terpene derivatives in $S$. cerevisiae can be achieved by optimization of endogenous pathways, employing CRISPR gene editing technology, modification of key enzymes and utilization of synthetic S. cerevisiae chromosome system (SCRaMbLE). More importantly, there are powerful tools such as computational biology, genomics and transcriptomics accompanied by synthetic biology to identify and manipulate novel terpenoid synthesis pathways in microbial cell factories for their green production. Advances in synthetic biology technology will promote to develop a fully automated robotic screening platform for high-throughput screening of engineered strains. Combination of different regulation strategies to design and improve the metabolic pathways in $S$. cerevisiae is expected to be an efficient approach over the traditional methods to sequester UA and its derivatives in future for further exploration. Although there is a long way to go to develop an economically feasible and easy method for UA, its rich pharmacological activity and great value potential are worthy of extensive exploration in many scientific fields.

\section{List of abbreviations}

S. cerevisiae $=$ Saccharomyces cerevisiae

$\mathrm{UA}=$ Ursolic acid

IPP $=$ Isopentenyl diphosphate

DMAPP $=$ Dimethylallyl diphosphate

FPP $=$ Farnesyl diphosphate

CYP450 = Cytochrome P450

$\mathrm{UGTs}=\mathrm{UDP}$-glycosyltransferases

$\alpha \mathrm{AS}=\alpha$-amyrin synthase

OSCs $=$ Oxidosqualene Cyclases

$\mathrm{ER}=$ Endoplasmic reticulum

\section{Declaration of Competing Interest}

The authors declare that they have no known competing financial interests or personal relationships that could have appeared to influence the work reported in this paper.

\section{Acknowledgements}

This work was supported by the National Key Research and Development Program of China (2018YFA0901800, 2019YFA0905700) and the National Natural Science Foundation of China (21736002, 21878021).

\section{Reference}

1 Vergara Martínez, V. M. et al. Methyl Jasmonate and Salicylic Acid Enhanced the Production of Ursolic and Oleanolic Acid in Callus Cultures of Lepechinia Caulescens. Pharmacognosy magazine13 , S886-s889, doi:10.4103/pm.pm_77_17 (2018).

2 Andre, C. M. et al. Anti-inflammatory procyanidins and triterpenes in 109 apple varieties. Journal of agricultural and food chemistry 60 , 10546-10554, doi:10.1021/jf302809k (2012).

3 Baricevic, D. et al. Topical anti-inflammatory activity of Salvia officinalis L. leaves: the relevance of ursolic acid.Journal of ethnopharmacology 75 , 125-132, doi:10.1016/s0378-8741(00)00396-2 (2001).

4 Saravanan, R., Viswanathan, P. \& Pugalendi, K. V. Protective effect of ursolic acid on ethanol-mediated experimental liver damage in rats.Life sciences 78, 713-718, doi:10.1016/j.lfs.2005.05.060 (2006). 
5 Ullevig, S. L., Zhao, Q., Zamora, D. \& Asmis, R. Ursolic acid protects diabetic mice against monocyte dysfunction and accelerated atherosclerosis. Atherosclerosis $\mathbf{2 1 9}$, 409-416, doi:10.1016/j.atherosclerosis.2011.06.013 (2011).

6 Kurek, A., Markowska, K., Grudniak, A. M., Janiszowska, W. \& Wolska, K. I. The effect of oleanolic and ursolic acids on the hemolytic properties and biofilm formation of Listeria monocytogenes. Polish journal of microbiology 63, 21-25 (2014).

7 Xiang, L. et al. A pentacyclic triterpene natural product, ursolic acid and its prodrug US597 inhibit targets within cell adhesion pathway and prevent cancer metastasis. Oncotarget 6 , 9295-9312, doi:10.18632/oncotarget.3261 (2015).

8 Rashid, S., Dar, B. A., Majeed, R., Hamid, A. \& Bhat, B. A. Synthesis and biological evaluation of ursolic acid-triazolyl derivatives as potential anti-cancer agents. European journal of medicinal chemistry $\mathbf{6 6}$ , 238-245, doi:10.1016/j.ejmech.2013.05.029 (2013).

9 Jin, H. et al. Ursolic acid-loaded chitosan nanoparticles induce potent anti-angiogenesis in tumor. Applied microbiology and biotechnology 100 , 6643-6652, doi:10.1007/s00253-016-7360-8 (2016).

10 Pateraki, I., Heskes, A. M. \& Hamberger, B. Cytochromes P450 for terpene functionalisation and metabolic engineering. Advances in biochemical engineering/biotechnology 148 , 107-139, doi:10.1007/10_2014_301 (2015).

11 Li, J. S., Wang, W. J., Sun, Y., Zhang, Y. H. \& Zheng, L. Ursolic acid inhibits the development of nonalcoholic fatty liver disease by attenuating endoplasmic reticulum stress. Food $\mathcal{E}$ function 6 , 1643-1651, doi:10.1039/c5fo00083a (2015).

12 Paramasivan, K. \& Mutturi, S. Progress in terpene synthesis strategies through engineering of Saccharomyces cerevisiae.Critical reviews in biotechnology 37 , 974-989, doi:10.1080/07388551.2017.1299679 (2017).

13 Xiao, H. \& Zhong, J. J. Production of Useful Terpenoids by Higher-Fungus Cell Factory and Synthetic Biology Approaches. Trends in biotechnology 34, 242-255, doi:10.1016/j.tibtech.2015.12.007 (2016).

$14 \mathrm{Lim}, \mathrm{S}$. W. et al. Simultaneous effect of ursolic acid and oleanolic acid on epidermal permeability barrier function and epidermal keratinocyte differentiation via peroxisome proliferator-activated receptor-alpha. The Journal of dermatology 34 ,625-634, doi:10.1111/j.1346-8138.2007.00344.x (2007).

$15 \mathrm{Yu}$, Z. et al. Pharmacokinetics in Vitro and in Vivo of Two Novel Prodrugs of Oleanolic Acid in Rats and Its Hepatoprotective Effects against Liver Injury Induced by CCl4. Molecular pharmaceutics 13, 1699-1710, doi:10.1021/acs.molpharmaceut.6b00129 (2016).

16 Pompei, R., Flore, O., Marccialis, M. A., Pani, A. \& Loddo, B. Glycyrrhizic acid inhibits virus growth and inactivates virus particles. Nature 281 , 689-690, doi:10.1038/281689a0 (1979).

17 Paddon, C. J. et al. High-level semi-synthetic production of the potent antimalarial artemisinin. Nature 496 , 528-532, doi:10.1038/nature12051 (2013).

18 Zhuang, Y. et al. Biosynthesis of plant-derived ginsenoside Rh2 in yeast via repurposing a key promiscuous microbial enzyme.Metabolic engineering 42, 25-32, doi:10.1016/j.ymben.2017.04.009 (2017).

19 Zhao, Y., Fan, J., Wang, C., Feng, X. \& Li, C. Enhancing oleanolic acid production in engineered Saccharomyces cerevisiae.Bioresource technology 257 , 339-343, doi:10.1016/j.biortech.2018.02.096 (2018).

20 Ma, T. et al. Lipid engineering combined with systematic metabolic engineering of Saccharomyces cerevisiae for high-yield production of lycopene. Metabolic engineering 52 , 134-142, doi:10.1016/j.ymben.2018.11.009 (2019).

21 Mancha-Ramirez, A. M. \& Slaga, T. J. Ursolic Acid and Chronic Disease: An Overview of UA's Effects On Prevention and Treatment of Obesity and Cancer. Advances in experimental medicine and biology $\mathbf{9 2 8}$, 
75-96, doi:10.1007/978-3-319-41334-1_4 (2016).

22 Hussain, H. et al. Ursolic acid derivatives for pharmaceutical use: a patent review (2012-2016). Expert opinion on therapeutic patents $\mathbf{2 7}$, 1061-1072, doi:10.1080/13543776.2017.1344219 (2017).

23 Checker, R. et al. Potent anti-inflammatory activity of ursolic acid, a triterpenoid antioxidant, is mediated through suppression of NF- $\varkappa$ B, AP-1 and NF-AT. PloS one 7 , e31318, doi:10.1371/journal.pone.0031318 (2012).

24 Kong, L. et al. Oleanolic acid and ursolic acid: novel hepatitis C virus antivirals that inhibit NS5B activity. Antiviral research 98 , 44-53, doi:10.1016/j.antiviral.2013.02.003 (2013).

25 Yim, E. K., Lee, M. J., Lee, K. H., Um, S. J. \& Park, J. S. Antiproliferative and antiviral mechanisms of ursolic acid and dexamethasone in cervical carcinoma cell lines. International journal of gynecological cancer : official journal of the International Gynecological Cancer Society 16 , 2023-2031, doi:10.1111/j.15251438.2006.00726.x (2006).

26 Kunkel, S. D. et al. Ursolic acid increases skeletal muscle and brown fat and decreases diet-induced obesity, glucose intolerance and fatty liver disease. PloS one $\mathbf{7}$, e39332, doi:10.1371/journal.pone.0039332 (2012).

27 Dong, X. et al. Downregulation of miR-21 is involved in direct actions of ursolic acid on the heart: implications for cardiac fibrosis and hypertrophy. Cardiovascular therapeutics 33 , 161-167, doi:10.1111/17555922.12125 (2015).

28 Meng, Y. et al. Ursolic Acid Induces Apoptosis of Prostate Cancer Cells via the PI3K/Akt/mTOR Pathway. The American journal of Chinese medicine 43, 1471-1486, doi:10.1142/s0192415x15500834 (2015).

29 Mendes, V. I. S., Bartholomeusz, G. A., Ayres, M., Gandhi, V. \& Salvador, J. A. R. Synthesis and cytotoxic activity of novel A-ring cleaved ursolic acid derivatives in human non-small cell lung cancer cells. European journal of medicinal chemistry 123 , 317-331, doi:10.1016/j.ejmech.2016.07.045 (2016).

30 Ramachandran, S. \& Prasad, N. R. Effect of ursolic acid, a triterpenoid antioxidant, on ultraviolet$\mathrm{B}$ radiation-induced cytotoxicity, lipid peroxidation and DNA damage in human lymphocytes. Chemicobiological interactions 176 , 99-107, doi:10.1016/j.cbi.2008.08.010 (2008).

31 Ramírez-Rodríguez, A. M., González-Ortiz, M., Martínez-Abundis, E. \& Acuña Ortega, N. Effect of Ursolic Acid on Metabolic Syndrome, Insulin Sensitivity, and Inflammation. Journal of medicinal food20 , 882-886, doi:10.1089/jmf.2017.0003 (2017).

$32 \mathrm{Li}, \mathrm{S}$. et al. Therapeutic role of ursolic acid on ameliorating hepatic steatosis and improving metabolic disorders in high-fat diet-induced non-alcoholic fatty liver disease rats. PloS one $\mathbf{9}$, e86724, doi:10.1371/journal.pone.0086724 (2014).

33 Xia, E. Q. et al. Microwave-assisted extraction of oleanolic acid and ursolic acid from Ligustrum lucidum Ait. International journal of molecular sciences 12, 5319-5329, doi:10.3390/ijms12085319 (2011).

34 Yoshida, M. et al. Antiproliferative constituents from Umbelliferae plants VII. Active triterpenes and rosmarinic acid from Centella asiatica. Biological \&3 pharmaceutical bulletin28, 173-175, doi:10.1248/bpb.28.173 (2005).

35 Papadopoulou, K., Melton, R. E., Leggett, M., Daniels, M. J. \& Osbourn, A. E. Compromised disease resistance in saponin-deficient plants. Proceedings of the National Academy of Sciences of the United States of America 96 , 12923-12928, doi:10.1073/pnas.96.22.12923 (1999).

36 Jäger, S., Trojan, H., Kopp, T., Laszczyk, M. N. \& Scheffler, A. Pentacyclic triterpene distribution in various plants - rich sources for a new group of multi-potent plant extracts. Molecules (Basel, Switzerland) 14, 2016-2031, doi:10.3390/molecules14062016 (2009). 
37 Brendolise, C. et al. An unusual plant triterpene synthase with predominant $\alpha$-amyrin-producing activity identified by characterizing oxidosqualene cyclases from Malus $\times$ domestica. The FEBS journal $\mathbf{2 7 8}, 2485-$ 2499, doi:10.1111/j.1742-4658.2011.08175.x (2011).

38 Farneti, B. et al. Is there room for improving the nutraceutical composition of apple? Journal of agricultural and food chemistry 63 , 2750-2759, doi:10.1021/acs.jafc.5b00291 (2015).

39 Guinda, A., Rada, M., Delgado, T. \& Castellano, J. M. Pentacyclic triterpenic acids from Argania spinosa. Eur. J. Lipid Sci. Technol. 113 , 231-237, doi:10.1002/ejlt.201000342 (2011).

40 Kowalski, R. Studies of selected plant raw materials as alternative sources of triterpenes of oleanolic and ursolic acid types. Journal of agricultural and food chemistry 55 ,656-662, doi:10.1021/jf0625858 (2007).

41 Wojciak-Kosior, M., Sowa, I., Kocjan, R. \& Nowak, R. Effect of different extraction techniques on quantification of oleanolic and ursolic acid in Lamii albi flos. Industrial Crops and Products44, 373-377, doi:10.1016/j.indcrop.2012.11.018 (2013).

$42 \mathrm{Fu}$, Q., Zhang, L., Cheng, N., Jia, M. \& Zhang, Y. Extraction optimization of oleanolic and ursolic acids from pomegranate (Punica granatum L.) flowers. Food and Bioproducts Processing 92 , 321-327, doi:10.1016/j.fbp.2012.12.006 (2014).

43 Sheng, H. \& Sun, H. Synthesis, biology and clinical significance of pentacyclic triterpenes: a multi-target approach to prevention and treatment of metabolic and vascular diseases. Natural product reports $\mathbf{2 8}$, 543-593, doi:10.1039/c0np00059k (2011).

44 Yin, M. C., Lin, M. C., Mong, M. C. \& Lin, C. Y. Bioavailability, distribution, and antioxidative effects of selected triterpenes in mice. Journal of agricultural and food chemistry $60,7697-7701$, doi:10.1021/jf302529x (2012).

45 Sultana, N. Clinically useful anticancer, antitumor, and antiwrinkle agent, ursolic acid and related derivatives as medicinally important natural product. Journal of enzyme inhibition and medicinal chemistry $\mathbf{2 6}$ , 616-642, doi:10.3109/14756366.2010.546793 (2011).

46 Mazumder, K., Tanaka, K. \& Fukase, K. Cytotoxic activity of ursolic acid derivatives obtained by isolation and oxidative derivatization.Molecules (Basel, Switzerland) 18, 8929-8944, doi:10.3390/molecules18088929 (2013).

$47 \mathrm{Liu}$, M. C. et al. Synthesis and cytotoxicity of novel ursolic acid derivatives containing an acyl piperazine moiety. European journal of medicinal chemistry 58 , 128-135, doi:10.1016/j.ejmech.2012.08.048 (2012).

48 Bai, K. K. et al. Synthesis and evaluation of ursolic acid derivatives as potent cytotoxic agents. Bioorganic 83 medicinal chemistry letters 22 , 2488-2493, doi:10.1016/j.bmcl.2012.02.009 (2012).

49 Chadalapaka, G., Jutooru, I., McAlees, A., Stefanac, T. \& Safe, S. Structure-dependent inhibition of bladder and pancreatic cancer cell growth by 2-substituted glycyrrhetinic and ursolic acid derivatives.Bioorganic 83 medicinal chemistry letters 18 , 2633-2639, doi:10.1016/j.bmcl.2008.03.031 (2008).

50 Shanmugam, M. K. et al. Oleanolic acid and its synthetic derivatives for the prevention and therapy of cancer: preclinical and clinical evidence. Cancer letters 346, 206-216, doi:10.1016/j.canlet.2014.01.016 (2014).

$51 \mathrm{Tu}, \mathrm{H} . \mathrm{Y}$. et al. Ursolic acid derivatives induce cell cycle arrest and apoptosis in NTUB1 cells associated with reactive oxygen species. Bioorganic $\mathscr{E}$ medicinal chemistry 17 , 7265-7274, doi:10.1016/j.bmc.2009.08.046 (2009).

52 Leal, A. S., Wang, R., Salvador, J. A. \& Jing, Y. Synthesis of novel ursolic acid heterocyclic derivatives with improved abilities of antiproliferation and induction of p53, p21waf1 and NOXA in pancreatic cancer cells. Bioorganic 85 medicinal chemistry 20 , 5774-5786, doi:10.1016/j.bmc.2012.08.010 (2012). 
$53 \mathrm{Liu}, \mathrm{X}$. T. et al. Cholestane and spirostane glycosides from the rhizomes of Dioscorea septemloba. Phytochemistry 69, 1411-1418, doi:10.1016/j.phytochem.2007.12.014 (2008).

54 Tian, J. et al. Dibenzo- $\alpha$-pyrones from the endophytic fungus Alternaria sp. Samif01: isolation, structure elucidation, and their antibacterial and antioxidant activities. Natural product research 31, 387-396, doi:10.1080/14786419.2016.1205052 (2017).

55 Zhang, L. H., Wang, H. W., Xu, J. Y., Li, J. \& Liu, L. A new Secondary metabolites of the crinoid (Comanthina schlegeli) associated fungus Alternaria brassicae 93. Natural product research30 , 2305-2310, doi:10.1080/14786419.2016.1166498 (2016).

56 Liu, D. L., Liu, Y., Qiu, F., Gao, Y. \& Zhang, J. Z. Biotransformation of oleanolic acid by Alternaria longipes and Penicillium adametzi. Journal of Asian natural products research13 , 160-167, doi:10.1080/10286020.2010.547028 (2011).

57 Zhang, J., Cheng, Z. H., Yu, B. Y., Cordell, G. A. \& Qiu, S. X. J. T. L. Novel biotransformation of pentacyclic triterpenoid acids by Nocardia sp. NRRL 5646. 46, 2337-2340 (2005).

58 Leipold, D. et al. Biosynthesis of ursolic acid derivatives by microbial metabolism of ursolic acid with Nocardia sp. strains - Proposal of new biosynthetic pathways. 45 , 1043-1051 (2010).

$59 \mathrm{Fu}, \mathrm{S}$. B. et al. Multihydroxylation of ursolic acid by Pestalotiopsis microspora isolated from the medicinal plant Huperzia serrata. Fitoterapia 82, 1057-1061, doi:10.1016/j.fitote.2011.06.009 (2011).

60 Ibrahim, A. et al. Microbial metabolism of biologically active secondary metabolites from Nerium oleander L. Chemical \& pharmaceutical bulletin 56 , 1253-1258, doi:10.1248/cpb.56.1253 (2008).

61 Huang, F. X. et al. Microbial transformation of ursolic acid by Syncephalastrum racemosum (Cohn) Schroter AS 3.264.Phytochemistry 82, 56-60, doi:10.1016/j.phytochem.2012.06.020 (2012).

62 Zhang, C. X., Ma, W. J., Liu, D. L., Jia, X. J. \& Zhao, Y. M. Biotransformation of ursolic acid by Alternaria longipes AS3.2875. Natural product research 32 , 536-543, doi:10.1080/14786419.2017.1327860 (2018).

63 Zhang, S.-S. et al. Three new triterpenoids transformed from ursolic acid by Mucor spinosus AS3.3450 and their cytotoxicity.Phytochemistry Letters 32, 33-37, doi:https://doi.org/10.1016/j.phytol.2019.04.019(2019).

64 Dai, Z., Liu, Y., Huang, L. \& Zhang, X. Production of miltiradiene by metabolically engineered Saccharomyces cerevisiae.Biotechnology and bioengineering 109, 2845-2853, doi:10.1002/bit.24547 (2012).

65 Liu, H., Fan, J., Wang, C., Li, C. \& Zhou, X. Enhanced $\beta$-Amyrin Synthesis in Saccharomyces cerevisiae by Coupling An Optimal Acetyl-CoA Supply Pathway. Journal of agricultural and food chemistry67 , 37233732, doi:10.1021/acs.jafc.9b00653 (2019).

66 Katabami, A. et al. Production of squalene by squalene synthases and their truncated mutants in Escherichia coli. Journal of bioscience and bioengineering 119, 165-171, doi:10.1016/j.jbiosc.2014.07.013 (2015).

67 Leonard, E. et al. Combining metabolic and protein engineering of a terpenoid biosynthetic pathway for overproduction and selectivity control. Proceedings of the National Academy of Sciences of the United States of America 107 , 13654-13659, doi:10.1073/pnas.1006138107 (2010).

68 Trikka, F. A. et al. Iterative carotenogenic screens identify combinations of yeast gene deletions that enhance sclareol production. Microbial cell factories 14,60, doi:10.1186/s12934-015-0246-0 (2015).

69 Westfall, P. J. et al. Production of amorphadiene in yeast, and its conversion to dihydroartemisinic acid, precursor to the antimalarial agent artemisinin. Proceedings of the National Academy of Sciences of the United States of America 109 , E111-118, doi:10.1073/pnas.1110740109 (2012). 
70 Zhao, J. et al. Dynamic control of ERG20 expression combined with minimized endogenous downstream metabolism contributes to the improvement of geraniol production in Saccharomyces cerevisiae.Microbial cell factories 16 , 17, doi:10.1186/s12934-017-0641-9 (2017).

71 Kushiro, T., Shibuya, M. \& Ebizuka, Y. Beta-amyrin synthase-cloning of oxidosqualene cyclase that catalyzes the formation of the most popular triterpene among higher plants. European journal of biochemistry 256 , 238-244, doi:10.1046/j.1432-1327.1998.2560238.x (1998).

$72 \mathrm{Hu}$, Y. et al. Metabolic engineering of Saccharomyces cerevisiae for production of germacrene A, a precursor of beta-elemene. Journal of industrial microbiology 83 biotechnology 44, 1065-1072, doi:10.1007/s10295017-1934-z (2017).

73 Czarnotta, E. et al. Fermentation and purification strategies for the production of betulinic acid and its lupane-type precursors in Saccharomyces cerevisiae. Biotechnology and bioengineering114, 2528-2538, doi:10.1002/bit.26377 (2017).

74 Klingenberg, M. Pigments of rat liver microsomes. Archives of biochemistry and biophysics 75 , 376-386, doi:10.1016/0003-9861(58)90436-3 (1958).

75 Dai, Z. et al. Identification of a novel cytochrome P450 enzyme that catalyzes the C-2 $\alpha$ hydroxylation of pentacyclic triterpenoids and its application in yeast cell factories.Metabolic engineering 51 , 70-78, doi:10.1016/j.ymben.2018.10.001 (2019).

76 Lamb, D. C. \& Waterman, M. R. Unusual properties of the cytochrome P450 superfamily. Philosophical transactions of the Royal Society of London. Series B, Biological sciences 368 , 20120434, doi:10.1098/rstb.2012.0434 (2013).

77 Chang, C. H. et al. The cysteine 703 to isoleucine or histidine mutation of the oxidosqualene-lanosterol cyclase from Saccharomyces cerevisiae generates an iridal-type triterpenoid.Biochimie 94, 2376-2381, doi:10.1016/j.biochi.2012.06.014 (2012).

$78 \mathrm{Wu}$, P. et al. Synthesis and Evaluation of Novel Triterpene Analogues of Ursolic Acid as Potential Antidiabetic Agent. PloS one 10 , e0138767, doi:10.1371/journal.pone.0138767 (2015).

$79 \mathrm{Lu}$, C., Zhang, C., Zhao, F., Li, D. \& Lu, W. Biosynthesis of Ursolic Acid and Oleanolic Acid in Saccharomyces cerevisiae. Aiche Journal 64 , 3794-3802, doi:10.1002/aic.16370 (2018).

80 Sun, W. et al. Novel trends for producing plant triterpenoids in yeast. Critical reviews in biotechnology 39 , 618-632, doi:10.1080/07388551.2019.1608503 (2019).

81 Zheng, X. et al. Characterisation of two oxidosqualene cyclases responsible for triterpenoid biosynthesis in Ilex asprella.International journal of molecular sciences 16 ,3564-3578, doi:10.3390/ijms16023564 (2015).

82 Zhang, G. et al. Refactoring -amyrin synthesis in Saccharomyces cerevisiae. Aiche Journal 61 , 3172-3179, doi:10.1002/aic.14950 (2015).

$83 \mathrm{Yu}$, Y. et al. Productive Amyrin Synthases for Efficient $\alpha$-Amyrin Synthesis in Engineered Saccharomyces cerevisiae. ACS synthetic biology 7 , 2391-2402, doi:10.1021/acssynbio.8b00176 (2018).

$84 \mathrm{Yu}, \mathrm{Y}$. et al. Engineering Saccharomyces cerevisiae for high yield production of $\alpha$-amyrin via synergistic remodeling of $\alpha$-amyrin synthase and expanding the storage pool. Metabolic engineering , doi:10.1016/j.ymben.2020.08.010 (2020).

85 Yasumoto, S., Fukushima, E. O., Seki, H. \& Muranaka, T. Novel triterpene oxidizing activity of Arabidopsis thaliana CYP716A subfamily enzymes. FEBS letters 590 , 533-540, doi:10.1002/1873-3468.12074 (2016).

86 Fukushima, E. O. et al. CYP716A subfamily members are multifunctional oxidases in triterpenoid biosynthesis. Plant $\&$ cell physiology 52, 2050-2061, doi:10.1093/pcp/pcr146 (2011). 
87 Moses, T. et al. OSC2 and CYP716A14v2 catalyze the biosynthesis of triterpenoids for the cuticle of aerial organs of Artemisia annua. The Plant cell 27, 286-301, doi:10.1105/tpc.114.134486 (2015).

88 Yasumoto, S., Seki, H., Shimizu, Y., Fukushima, E. O. \& Muranaka, T. Functional Characterization of CYP716 Family P450 Enzymes in Triterpenoid Biosynthesis in Tomato. Frontiers in plant science 8 , 21, doi:10.3389/fpls.2017.00021 (2017).

89 Suzuki, H. et al. Comparative analysis of CYP716A subfamily enzymes for the heterologous production of C-28 oxidized triterpenoids in transgenic yeast. Plant biotechnology (Tokyo, Japan)35 , 131-139, doi:10.5511/plantbiotechnology.18.0416a (2018).

90 Han, J. Y., Kim, M. J., Ban, Y. W., Hwang, H. S. \& Choi, Y. E. The involvement of $\beta$-amyrin 28 -oxidase (CYP716A52v2) in oleanane-type ginsenoside biosynthesis in Panax ginseng. Plant $\&$ cell physiology $\mathbf{5 4}$, 2034-2046, doi:10.1093/pcp/pct141 (2013).

91 Khakimov, B. et al. Identification and genome organization of saponin pathway genes from a wild crucifer, and their use for transient production of saponins in Nicotiana benthamiana. The Plant journal : for cell and molecular biology 84, 478-490, doi:10.1111/tpj.13012 (2015).

92 Miettinen, K. et al. The ancient CYP716 family is a major contributor to the diversification of eudicot triterpenoid biosynthesis.Nature communications 8 , 14153, doi:10.1038/ncomms14153 (2017).

93 Andre, C. M. et al. Multifunctional oxidosqualene cyclases and cytochrome P450 involved in the biosynthesis of apple fruit triterpenic acids. The New phytologist 211 , 1279-1294, doi:10.1111/nph.13996 (2016).

94 Tamura, K. et al. CYP716A179 functions as a triterpene C-28 oxidase in tissue-cultured stolons of Glycyrrhiza uralensis. Plant cell reports 36 , 437-445, doi:10.1007/s00299-016-2092-x (2017).

95 Misra, R. C. et al. Two CYP716A subfamily cytochrome P450 monooxygenases of sweet basil play similar but nonredundant roles in ursane- and oleanane-type pentacyclic triterpene biosynthesis. The New phytologist 214, 706-720, doi:10.1111/nph.14412 (2017).

96 Sandeep, Misra, R. C., Chanotiya, C. S., Mukhopadhyay, P. \& Ghosh, S. Oxidosqualene cyclase and CYP716 enzymes contribute to triterpene structural diversity in the medicinal tree banaba. The New phytologist 222, 408-424, doi:10.1111/nph.15606 (2019).

97 Huang, L. et al. Molecular characterization of the pentacyclic triterpenoid biosynthetic pathway in Catharanthus roseus. Planta236 , 1571-1581, doi:10.1007/s00425-012-1712-0 (2012).

98 Farhi, M. et al. Harnessing yeast subcellular compartments for the production of plant terpenoids. Metabolic engineering13 , 474-481, doi:10.1016/j.ymben.2011.05.001 (2011).

99 Arendt, P. et al. An endoplasmic reticulum-engineered yeast platform for overproduction of triterpenoids. Metabolic engineering 40 , 165-175, doi:10.1016/j.ymben.2017.02.007 (2017).

100 Nakamura, M. et al. Transcriptome sequencing and identification of cytochrome P450 monooxygenases involved in the biosynthesis of maslinic acid and corosolic acid in Avicennia marina.Plant biotechnology (Tokyo, Japan) 35, 341-348, doi:10.5511/plantbiotechnology.18.0810a (2018).

$101 \mathrm{Xu}, \mathrm{Y}$. et al. Blocking inhibition to YAP by ActinomycinD enhances anti-tumor efficacy of Corosolic acid in treating liver cancer.Cellular signalling 29 , 209-217, doi:10.1016/j.cellsig.2016.11.001 (2017).

$102 \mathrm{Ji}$, X. et al. Identification of $\alpha$-Amyrin 28-Carboxylase and Glycosyltransferase From Ilex asprella and Production of Ursolic Acid 28-O- $\beta$-D-Glucopyranoside in Engineered Yeast. Frontiers in plant science 11 , 612, doi:10.3389/fpls.2020.00612 (2020). 